\title{
Laws are conditionals
}

\author{
Toby Friend ${ }^{1}$
}

Received: 24 April 2015 / Accepted: 23 November 2015 / Published online: 14 January 2016 (C) The Author(s) 2016. This article is published with open access at Springerlink.com

\begin{abstract}
The ubiquitous schema 'All Fs are Gs' dominates much philosophical discussion on laws but rarely is it shown how actual laws mentioned and used in science are supposed to fit it. After consideration of a variety of laws, including those obviously conditional and those superficially not conditional (such as equations), I argue that we have good reason to support the traditional interpretation of laws as conditionals of some quantified form with a single object variable. I show how this conclusion impacts on the status of 'system laws' and the significance of having only one object variable with regard to the relationship between laws and causal relations.
\end{abstract}

Keywords Laws nature $\cdot$ Logical form · Conditionals $\cdot$ System $\cdot$ Equations

\section{Introduction}

An often assumed, but rarely argued for, view of laws of nature is that they are quantified conditionals. The ubiquitous schema 'All Fs are Gs' dominates much philosophical discussion on laws but rarely is it shown how actual laws mentioned and used in science are supposed to fit it. Instead, what can seem embarrassingly toy examples like 'all ravens are black' are employed for discussion. Ignoring the complexity of real cases has some value, but it also comes at a price, and today there is increasing literature arguing that laws have been misrepresented: either we have been wrong about their logical form or else we are misguided in supposing them to be important features of science.

Toby Friend

tobythomasfriend@gmail.com

1 Science and Technology Studies, University College London, London, UK 
My project here will be to offer some argument for the view that laws do indeed have a quantified conditional form. ${ }^{1}$ In Section 2 I will give some motivation for why an investigation of the logical form of laws in general should be of interest and say something about my methodology for picking examples for discussion. In Section 3 I focus the discussion on laws' conditionality and mention how it impacts on wider debates in the philosophy of science. The central part of the paper begins with 4 where I introduce a number of laws, some of which clearly are conditionals and some recalcitrant cases which are not obviously so, including equations. Starting, in 4.2 with an examination of the logical form of the laws represented as equations, then extending the reasoning to the miscellaneous class in 4.3, I argue that we have good reason to interpret all the recalcitrant cases as representing information which is ultimately conditional in form with a single object variable. In Section 5, I provide further argument building on the previous section for why we should think all laws describe the behaviour of systems which satisfy antecedent conditions (i.e. are conditionals). I conclude, in Section 6 with some summary remarks and comments on two further discussions informed by the preceding arguments, one regarding the distinction between 'system laws' and 'fundamental laws', another comparing the view that laws are conditionals with only one object variable with a broader conception of laws as conditionals of some type. I mention how this latter discussion impacts on the debate over law-based analyses of token causation.

\section{Logical form vs analysis}

The laws I have in mind for discussion are those Nancy Cartwright calls 'associative laws' which 'tell how often two [or more] qualities or quantities are co-associated' and are to be distinguished from causal laws which "have the word "cause"-or some causal surrogate, right in them' $(1979,419)$. Examples are Newton's law of gravitation, Farr's law of epidemics, Boyle's gas law, the law of (chemical compounds') definite proportions and the law of (geological) superposition. My purpose is to defend the view that laws of association (simply called 'laws' from now on, unless otherwise specified) have a quantified conditional form, although I will stop short of specifying the exact logic of the quantifier and the conditional. ${ }^{2}$

Why enquire into the logical form of laws at all rather than what laws are about? For a number of reasons, but ultimately because I believe it is a necessary yet distinct first step towards a comprehensive analysis of laws. To parrot Davidson, who carried out an analogous enquiry regarding singular causal statements, 'this question [of logical form] is more modest than the question how we know such statements are true, and the question whether they can be analyzed in terms of, say, constant conjunction. The request for logical form is modest because it is answered when we have

\footnotetext{
${ }^{1}$ I will not, therefore, be considering the interpretation of 'law' as denoting something non-linguistic, such as a contingent second order relation of necessitation (proposed, e.g., in Armstrong 1983, 1997). Laws, under the present discussion, are interpreted statements (potentially 'propositions') which have logical form.

${ }^{2}$ Thank you to an anonymous referee for pointing out the significance of this qualification.
} 
identified the logical or grammatical roles of the words $[\ldots]$ in the sentences under scrutiny. It goes beyond this to define, analyse, or set down axioms governing, particular words or expressions' $(1967,1)$. Thus, by investigating the logical form of laws, we can (at least try to) avoid many of the deep epistemological, conceptual and ontological issues which arise when we investigate what they are about directly. Yet arguably, such a project is also a necessary preliminary for these further investigations. For providing the logical form of a sentence is an essential aspect of providing truth-conditions for that sentence and it is difficult to see how we could hope to complete a non-circular conceptual, epistemological or ontological analysis of laws without also saying what the truth-conditions of those laws are.

While I hope this goes some way to persuade the hesitant about the project, I should say something more about what the question of logical form does not do. Perhaps the central question of laws of nature is what distinguishes them from accidental truths. If some proposition $p$ is a law, we can infer that in not too distant counterfactual scenarios it would still be that $p$, i.e. $p$ is modally robust. ${ }^{3}$ This is not the case with many other propositions. But insofar as our enquiry into the logical form of an expression does not explore its modality, our conclusions cannot be expected to provide an account of this aspect of laws.

Throughout the discussion, I will be drawing on examples from science. Picking examples is a tricky business. Philosophers tend to have their favourite laws (often either toy examples or else the most austere laws of physics) and disregard many of the statements called 'law' across the whole of the sciences. My approach is consciously opposed to this: cast the net wide and remove unwanted catch afterwards. For case-studies, I attempt to pick a wide variety of statements referred to as 'law' from a variety of sciences. Philosophers (and some scientists) may balk at some of the examples, if they are obviously ridden with counter-instances, not general enough, not from an austere enough science, etc. While I am happy to accept that a final (ontological, epistemological, conceptual) analysis of laws may see fit to disregard some of my examples, my feeling is that to make such a move before the current preliminary project has returned some conclusions would be arbitrary or a result of unjustified bias.

Having said that, there are clearly some statements we call laws which presumably scientists themselves would disregard as important objects of philosophical or scientific enquiry. For instance,

Bode's law: All planets 1 to $m$ orbit the sun in an ellipse with a semi-major axis $\alpha=0.3 \times 2^{n}+0.4$ astronomical units, where $n=-\infty$ when $m=1$ and $n=m-2$ for all $m>1$.

Bode's law (sometimes called 'Bode's rule') is largely discredited from capturing any generality worth investigating. First, it is false. Many planets do not satisfy its prediction. Second, there are no known plausible explanations as to why such a law

\footnotetext{
${ }^{3}$ For a more precise characterisation of laws' modal profile, we might follow Lange $(2000,2008,2009)$ in saying that $p$ is a law if and only if $p$ is not a logical truth and for any counterfactual suppositions $q, r, \ldots$, consistent with the set of laws, the subjunctive conditionals $q \square \rightarrow p, r \square \rightarrow(q \square \rightarrow p), \ldots$ are true.
} 
would hold even approximately. The fact that Bode's law is still called a law by scientists may seem to indicate that going by scientists' attributions of the title is not a good way to decide what lawlike statements are worthy of philosophical investigation.

While I take it that there may be numerous such statements in science, I will assume its fairly clear which they are. In what follows I will choose examples partly based on what scientists call laws, but also filtering for those which are actually employed in scientific practice. Bode's law, so far as I know, is not employed in that capacity and never was to any significant degree.

\section{What's the question and why ask it?}

Laws have long been described in philosophical literature as conditionals stating that if anything has some quality, then it has another. Typically, this is more formally represented by the famous, and for some infamous, schema, ${ }^{4}$

$$
\forall x(F x \rightarrow G x)
$$

Schema (1) characterises the logical form of laws as universally quantified conditionals where $F$ and $G$ are replaced by predicate clauses. Traditionally, the domain is understood broadly and the conditional ' $\rightarrow$ ' is taken to be material..$^{5}$

In some rendering or other, (1) is commonplace in the philosophical literature on laws of nature. I don't know who was the first to provide this schema for lawstatements, but it is often the go-to shorthand for philosophers' general arguments about them, purporting to capture the most famous of philosophers' examples of pseudo-law as 'all ravens are black', 'all swans are white', 'all emeralds are green'. But though ubiquitous, the schema has become the focus of some (not always unjustified) ridicule from scientists and more practice-focused philosophers of science. The three examples just provided, for example, are not laws in any sense in which a working scientist would consider. Schema (1), we might think, is at best an oversimplified philosopher's caricature. Maudlin (2007), for instance, has complained that,

The fundamental law of Newtonian mechanics, the mathematical consequence of Newton's first two laws, is $\mathbf{F}=m \mathbf{a}$ or $\mathbf{F}=m d^{2} \mathbf{x} / d t^{2}$ or, most precisely, $\mathbf{F}=m d(m \mathbf{v}) / d t$ [sic]. ${ }^{6}$ The fundamental law of non-relativistic quantum

\footnotetext{
${ }^{4}$ Some past instances are Hempel and Oppenheim (1948), Sellars (1948), Kneale (1950), Ayer (1999 [1956]), Fodor (1991), and Harré (1993). More recently, the schema can be found in, e.g., Cohen and Callender (2009), Nickel (2010), Beebee (2011), and Carroll (2012).

${ }^{5} \mathrm{By}$ arguing that we should schematise laws in the form of (1), I do not mean to deny that there are distinct logical forms which are equivalent to them, for example, that obtained by conjoining a law with a logical truth. The discussion which follows aims to make perspicuous a natural way of rendering laws which reveals the particular type of inference they license, viz. from a type of system to a type of behaviour of that system.

${ }^{6}$ There are too many variables for mass here. I imagine Maudlin means that the more precise rendering is $\mathbf{F}=d(m \mathbf{v}) / d t$.
} 
mechanics, Schrödinger's equation, is $i \hbar \partial \Psi / \partial t=\hat{H} \Psi$. No doubt these can be tortured into a form similar to $\forall x(F x \rightarrow G x)$, but it is hard to see what the purpose of the exercise would be. $(2007,11)$

Certainly, it is not immediately obvious why we should expect equations-the mainstay of representations of law in many areas of science not least physics- to be representing information most appropriately captured by (1). But if I am right, there must be some way to do so. Furthermore, if the defence provided is supposed to count for a significant proportion of laws, as well as being informative to wider philosophical projects regarding laws of nature, it had better not be too torturous a procedure. It is clear, however, that there has been almost no justification of the repeated use of this schema to represent laws in the philosophy of science literature, and since extant ubiquity doesn't entail correctness, the time has come to provide some.

There are a number of features of (1) which require defending. One feature of logical renderings of laws in general which has invited a lot of criticism and discussion is their universality or strictness (see, for a brief sample, Anscombe 1971; Cartwright 1979, 1989; Harré 1993; Giere 1995; Hüttemann 2007). As a consequence, the use of the universal quantifier ' $\forall$ ' in (1) is a legitimate point of concern (see Fodor 1991; Pietroski and Rey 1995; Schurz 2002; Spohn 2002, Nickel 2010; Reutlinger 2011; Hüttemann 2014, for some responses). Unfortunately, limitations on space prohibit me from developing my position on this aspect of schema (1) here. The project I have in mind for the current paper is to argue for what comes after the quantifier: the conditional with antecedent and consequent predications of the same variable. Given the foregoing, my claim 'laws are conditionals' should be interpreted from hereon as a shorthand for the claim that laws are conditionals of some quantified form with a single bound variable. However, given that this project would be far too difficult without explicitly adopting some quantifier to bind the variables in examples, I will proceed as though the (default) universal quantifier is appropriate, in the knowledge that eventual further discussion of its employment will be necessary.

My argument for the conditional feature of the schema of laws aims to show that a conditional is implicit in our very understanding of laws, even when they do not appear to explicitly have that form. But before providing such an argument, I must pause again to further qualify the project. For even granting that laws are conditionals, we may yet wonder what conditionals they are. Typically, when laws have been represented with (1) the conditional is interpreted as material. Hence, the conditional is truth-functional: it is true so long as nothing which satisfies the antecedent also fails to satisfy the consequent. This interpretation has raised concern even with those sold on the conditionality of laws. When the ' $\rightarrow$ ' is understood as a material conditional, schematising laws with (1) renders them structurally equivalent to trivial universal conditionals which are true because nothing satisfies the antecedent (as in 'all unicorns are purple') or true because everything satisfies the consequent (as in 'all electrons are actual'). Furthermore, all material conditionals are logically equivalent to their contraposition, which intuitively can seem less worthy of the title 'law'. Such characteristics might seem to indicate the material conditional as being too liberal to capture the form of laws of nature. 
Other interpretations of the conditional in lawlike statements have been provided which are more restrictive. For example, we may wish to treat laws as inferences about kinds (proposed in Lowe 2009), or as inferences about abstract models (proposed in Giere 1988), or as an assertion of the existence of a relation of necessitation between two first-order universals (Armstrong 1983, thought laws were to be identified with such existences). There have also been more logically exotic suggestions, that laws should be understood as non-truth-functional conditionals (along the lines suggested by Stalnaker 1968) or under some suppositional theory (suggested, for instance, in Mackie 1973, ch. 4). Still other renderings employ an even more liberal conditional than the material conditional, such as those which treat laws as generics in which the inference from antecedent to consequent is qualified as holding only in 'normal' circumstances (e.g. Schurz 2002; Drewery 2006; Nickel 2010).

I have yet to be persuaded that the material conditional is not appropriate for rendering laws, although I will refrain from arguing this here. For those of us who believe laws to have the form of some conditional or other, a more pressing concern is to justify just that, if we can, before getting bogged down in the finer details of the logic. Of course, it might be the opinion of some who believe that laws are conditionals that there is no way to show this until we have the logic right. I disagree, and although I will write with the material conditional in mind, I intend my comments to be persuasive of the conclusion that laws are conditionals regardless of which particular version of conditional this may turn out to be.

This conclusion is significant for many areas of discussion in philosophy of science (e.g. discussions about the status of laws' universality and their determinism). Whilst I do not have space to elaborate on most of these areas, I will endeavour to say something a little more developed regarding two points of contact with wider literature. The first is represented by a number of authors (Beatty 1995; Rosenberg 2001; Schurz 2002; Reutlinger 2011) who could be interpreted as seeking to distinguish laws which do express such conditionals from those more significant laws which don't. Since all laws are conditionals according to the arguments put forward in this paper, the view according to this interpretation of these authors is considered and rejected in Section 6.1.

The second point of contact regards the specific fact that the conditional form I am defending has antecedent and consequent as predications of the same variable. Some authors (e.g. Hempel 1965; Pietroski and Rey 1995) have chosen to characterise laws as conditionals with a broader logical form than instances of schema (1) have. I will show in Section 6.2 why the arguments presented here tell against these characterisations and indicate some consequences for the debate on laws' relationship with causation.

\section{Are any laws conditionals?}

Certainly some of the statements titled 'law' in science very clearly have the form of a conditional. Take the following examples (many of my examples throughout are drawn from Lange 2000, 30-33), 
Farr's law: All epidemics follow a positive, symmetrical curve of infected population over time.

Werner's law: Geological strata are never inverted.

Wallace's law: Every species has come into existence coincident both in time and space with a preexisting closely allied species.

Although none of the above expressions contain explicit 'if' and 'then' clauses, they can clearly be understood as telling us that for all things, if they are of a certain type, then they will be a certain way, i.e. there is an antecedent comprising a classificatory predication of the kind of system the law is about (e.g. an epidemic, a stratum, a species), and a consequent comprising a predication of the kind of behaviour (in a broad sense) systems of the antecedent type are expected to exhibit (e.g. a certain dynamics of size over time, a directionality with respect to the centre of the earth, a relationship with other species at certain times in the species' history). They can thus be rendered into the form of (1), e.g.

$$
\forall x(\operatorname{Geological}-\operatorname{stratum}(x) \rightarrow \operatorname{not}-\operatorname{inverted}(x))
$$

\subsection{Recalcitrant Laws}

Despite laws like the above, which clearly are available to a conditional interpretation, many do not look, at first glance, to have this form at all. The above quote from Maudlin draws our attention to two laws often represented by equations. Consider also the following (allowing the variables to be defined in their usual way):

$$
\text { Ideal gas law: } P V=n R T \text {. }
$$

Newton's law of gravitation: $F_{M}=F_{m}=G \frac{M m}{r^{2}}$.

$$
\text { Ohm's Law: } V=I R \text {. }
$$

Yoda's power law: $W=C \rho^{-3 / 2}$.

$$
\text { Gauss's law: } \int_{S} D \cdot d S=\iiint_{V} \rho \cdot d v \text {. }
$$

Moreover, there are laws which are neither characteristically represented by equations nor in the form of a conditional, such as,

Gresham's law: Overvalued currency replaces undervalued currency in economic circulation. ${ }^{7}$

\footnotetext{
${ }^{7}$ Here 'overvalued/undervalued currency' indicates the ratio of a currency's 'commodity' value compared to its 'face value'. For example, if goods can be bought for in both paper money and gold, the law implies that paper money will replace the gold in circulation over time since, unlike gold, its commodity value is relatively low compared with its face value.
} 
Buys Ballot's law: Low pressure in the northern [southern] hemisphere is on the left [right] hand when facing downwind.

Faraday's first law of electrolysis: The mass of an element liberated or deposited on entering into reaction at an electrode during electrolysis is proportional to the quantity of electricity passing through it.

Granted, we may feel uncomfortable admitting all the above into a philosophical discussion of laws of nature. After all, Gresham's law seems dangerously close to counting as a causal law (in Cartwright's sense); this would be more perspicuous if, as is often the case, 'drives out' is substituted for 'replaces'. Also, Buys Ballot's law may seem unduly singular to count as an instance of a law, being implicitly restricted in application to Earth. Furthermore, we might expect that many of the laws above admit of a mechanistic explanation or reduction to simpler laws. For instance, we explain the Buys Ballot law in terms of the Coriolis effect. It is conceivable that certain metaphysical motivations might lead one to demand the full generality and non-reducibility of laws, in which case one might complain that some or all the above statements fail to count as proper Laws of Nature. But for the moment the project will be to see how far we get without taking action on the basis of such complaints. I will be assuming that the above examples are all as good examples of scientific law (of association) as each other, although I will be arguing that they are represented in a misleading way.

If the reader is happy to follow my lead in treating the above non-conditionally represented statements as at least prima facie examples of laws, why should we think that laws ultimately have the form of a conditional? My argument is really very simple. These laws describe how things behave. But none describe how everything behaves, so the characterisation of behaviour must be restricted to some antecedent system-type. Hence the conditional. While I take this brief summary to sound plausible up to a point, it clearly needs some more developed exegesis. The argument is perhaps most forcefully applied to those laws typically represented by (and misinterpreted as) equations. So I recommend we start by looking there.

\subsection{Equations and their laws}

What do the symbols either side of the '=' sign in the ideal gas law represent? Certainly not properties. If the symbols named properties, the expression would appear like an ill-formed identity (since identities are dyadic), not an equation. Anyway, it is hard to conceive how the gas constant $R$ could be a property, after all what kind of a thing would instantiate it? $P, V, n$ and $T$ are not obviously properties either, because they are variables taking any positive real number (incl. 0) as their values. However, the meaning of the gas law cannot be simply the algebraic statement that the product of any two numbers equals the product of a constant multiplied by two other numbers - that is a trivial truth of mathematics! And we know this cannot be the case since the variables in the ideal gas law, unlike purely mathematical equations, are associated with scientific units, e.g. Newtons-per-meter-squared $\left(\mathrm{Nm}^{-2}\right)$ for pressure, and degrees-Kelvin $(K)$ for temperature. 
A step in the right direction is to understand $P V=n R T$ as describing a group of functional relationships over property-types understood as sets of possible values for pressure, volume, quantity and temperature. For example, one function included in the group would be that which takes any value in a set of pressures as input and outputs some value in a set of temperatures. But this cannot be the whole story because functions, unlike laws, are not the sorts of things which can be truth-evaluable: a function is an operation not a statement. We could understand the equation as indicating a statement of the form 'there exists such a function between sets of pressures and sets of temperatures with the following results', but this again is an uninteresting fact, since there exist infinite such functions. If laws were the equations themselves, it is hard to see either what they represent or how they could be useful.

What is implicit in the formulation of the laws expressed as equations is the added clause that the group of functional relationships described by them are true of things. The set of temperatures, pressures, volumes and quantities of substance are those belonging to one and the same entity to which the equation may be applied. And the relationships described don't just exist in some abstract sense, but reflect the relations between the actual specific pressure, volume, temperature and quantity of such an entity at some moment in time. This suggests that the information bound up in equation $P V=n R T$ is something to be predicated of an entity, something an entity can satisfy or not. In general, we may understand equations to indicate a certain high-order behavioural property which entities have when and only when a specific group of relations determined by the equation holds between various (usually quantifiable) lower-order properties of that entity.

In the case of the ideal gas equation the relevant behavioural property is instantiated by something so long as the functions described by the equation map from actually instantiated properties to actually instantiated properties. Consider the pressure-function $P(x, t)$, the volume-function $V(x, t)$, the temperature-function $T(x, t)$ and the quantity-function $n(x, t)$, each of which take as inputs an entity and time and outputs a real number. This output defines the specific lower-order properties of the respective property-types pressure, volume, temperature and quantity of substance. The relevant higher-order behavioural predicate for the ideal gas law would be one which is satisfied by entity $g$ at $t_{1}$ only if the compound productfunction $P \times V$ which takes as inputs the outputs of the $P(x, t)$ and $V(x, t)$ functions and outputs the same value when $x=g$ and $t=t_{1}$ as does the compound productfunction $n \times R \times T$ which takes as inputs the outputs of the $T(x, t), n(x, t)$ functions and the constant $R$.

So the equations employed to represent laws are informative of the behaviour of things. But laws cannot have the form $B x$ where $x$ is a variable ranging over entities and $B$ is a behavioural predicate indicating that whatever satisfies it bears certain relations. This is simply ungrammatical. Laws are supposed to be general, so a plausible binding of the variable is with the universal quantifier: $\forall x(B x){ }^{8}$ However, it

\footnotetext{
${ }^{8}$ It is at this stage that accounts such as Armstrong's (1983), Giere's (1988) and Lowe's (2009) diverge from that developed here. I encourage the reader sympathetic to these treatments of law to continue with the present argument substituting the universal quantifier and domain of objects for their favoured quantification and domain.
} 
is certainly not the case that scientists use the ideal gas law, or Newton's, Ohm's or Yoda's law to say that everything bears these relationships between their properties. Concerns of absolute generality aside, to be the sort of thing which can have its components related in the way described by the behavioural predicate, that thing must at least have instances of those property-types, and many entities don't have any of them. After all, what's the pressure of a government, or the volume of a thought? But even when granting only the systems which have the relevant property-instances, the equations (or other functional relationships) described by the behavioural predicates are not indiscriminately attributed by scientists to all such systems. An apple has an internal pressure, volume, quantity of substance and a temperature, but we don't expect to use the equation $P V=n R T$ to describe its behaviour. Similarly, a lamppost has weight and density, but one doesn't ask what the respective constant is in order to predict its behaviour with the equation in Yoda's power law.

Assuming we want to preserve truth as best we can while retaining generality, laws like the ideal gas law, Ohm's law and Yoda's power law will need to be captured by a more qualified schema than $\forall x(B x)$ for a relatively broad domain. ${ }^{9}$ Note, this is a different problem from that which arises when we consider counterinstances to the law. One major complaint with treating laws as universally quantified is that many of them would thereby appear falsified by the existence of counterinstances. I have eschewed finding a solution to this problem in the present discussion (so I will not be settling whether or not to keep the quantifier and explain away the counterinstances or else modify the quantifier somehow). However, the present issue does not concern these kinds of counterinstances. Countries, thoughts and apples are not countersinstances to the ideal gas law, since they were never even in the running for candidate systems to describe with it. We might, therefore, understand the issue presently under discussion as that of explaining why these examples are not even in the running for being counterinstances to the ideal gas law (in contrast to, for example, a particular gas in Boyle's air-pump).

There appear to be two plausible options to qualify the general schema in order to make sense of this fact. One option is to understand the domain quantified over by the universal quantifier as restricted to only those things scientists are wont to ascribe the behavioural predicate to. In the case of the ideal gas law, for instance, the restriction would be to ideal gases (or those gases which may be appropriately modelled as an ideal gas). Such a response would be similar to that often made in order to make sense of utterances like that of Yogi Berra when he said that "nobody goes there anymore, it's too crowded." Without some suitable difference in domain restriction of Yogi's first clause from that of the second this sentence appears contradictory.

But is domain restriction sufficient to capture what is going on in the expression of laws? I will offer a few suggestive points to think not. ${ }^{10}$ First, laws are supposed

\footnotetext{
${ }^{9}$ Assuming truth means ignoring interpretations like that of Bach (1994), who offers a pragmatic reading of general statements for which there are counterinstances as strictly false, but where expressions of them nonetheless manage to convey some true proposition.

${ }^{10}$ That is not to say that domain restriction to less-than-universal contexts is not employed at all in the expression of laws. As Maudlin $(2007,12)$ points out, it does not appear to be contradictory to assert that some law governs physical states around here, or for the last 10 billion years, and we might deem it
} 
to be re-statable across a variety of contexts and, assuming they don't change (see Lange 2008, for a discussion), across time too. Laws are repeated in textbooks and science classes, they are derived, explained, invoked for calculation, written down by students. But further restrictions on the domain of the sort we have been considering are typically considered to be determined by contextual parameters (Stanley and Szabo 2000). For example, if the domain of quantification is restricted more in an utterance of "everything is gone" when uttered on return to my burgled home than when uttered at the end of the universe, then the extra restriction (e.g. to the things in my house or in my jewelry box) will be provided by the context rather than by linguistic features of the utterance. Laws are not the sort of expressions which should be susceptible to so much influence from context. An electrician, for instance, wants Ohm's law to be about the same things from one situation to another. Otherwise, it is hard to see why she should hope to apply all her understanding from case to case.

Second, it simply does not seem to be the case that laws are expressed in such a way that they ascribe a certain behaviour to everything and expect domain restriction to do all the work of cutting out unwanted denotation. Invariably, when the terms $P, V, n$, and $T$ are defined, in relation to an application of the equation $P V=n R T$, they are defined, respectively, as the pressure/volume/quantity-ofsubstance/temperature of a specific gas in a cavity. In other words, the terms represent property-variables of a certain type of thing: an ideal gas. Similarly, when the terms in $V=I R$ are defined, $V$ is defined as the potential difference measured across a conductor, $I$ as the current through the same conductor, and $R$ as the resistance of that conductor. Here, the property-variables are of one and the same electrical conductor.

The explicit reference to what type of thing the variables are supposed to be of suggests another way to qualify the schema. This option is to keep the domain broad and condition the ascription of behaviour on the satisfaction of some further predicate $S$, e.g. 'being an ideal gas' or 'being an electrical conductor', which picks out a kind of system, from which the behaviour can be lawfully inferred. To take this option is to understand laws as conditionals of the form $\forall x(S x \rightarrow B x)$. For example, what is missing in the representation of the ideal gas law as an unrestricted generalised predication of behaviour, according to the interpretation prescribed by this option, is the added clause that the relationships entailed by the equation in the behavioural predicate exist between the pressure, volume and temperature of any ideal gas, i.e.,

Ideal Gas Law*: If a system is an ideal gas then its temperature, pressure, quantity of substance and its volume will fix the values in the equation: $P V=n R T$

A proper understanding of the utility of the expression $P V=n R T$ requires us to understand it in the context of Ideal Gas Law*, i.e. a conditional. The law predicates a type of behaviour of something if we can predicate something else of that thing: that it is an instance of a certain type of system (e.g. an ideal gas). The same goes for the other equations often passed off as themselves laws. Although, as Maudlin

appropriate to attribute such restrictions to limitations on the domain rather than any explicit antecedent condition. However, my present criticism is with the idea that domain restriction is sufficient to limit application of the behaviour-predicate. 
says, it would be torturous to put the equations into the form of Eq. (1), this does not mean that laws themselves don't naturally take this form. For instance, Ohm's law of resistance is not an equation, but a conditional which states that all components with a constant value of resistance in an electronic circuit will experience a voltageloss and current distributed in accordance with the equation $V=I R$; similarly, Yoda's power law is not an equation but a conditional which states that all plant seedlings in a single sewn area will grow with their properties of dry-weight and density remaining related by the equation $W=C \rho^{-3 / 2}$. Newton's law of gravitation is a conditional which states that all two-mass systems will experience gravitational components of force proportional to the product of each other's mass according to the equation $F_{M}=F_{m}=G \frac{M m}{r^{2}}$. Gauss's law is a conditional stating that all closed surfaces are such that the flux density across them $\left(\int_{S} D . d S\right)$ is equal to the charge contained within $\left(\iiint_{V} \rho . d v\right)$.

This discussion of laws which are represented by (and often interpreted as) equations has resulted in a number of important conclusions. First, to understand an equation as something scientifically informative, it is necessary to interpret it as describing a set of functional criteria relating something's physical properties. This observation suggested the introduction of the idea of a 'behavioural-predicate' corresponding to the equation which is defined to be satisfied by an entity if and only if its physical properties are related by that set. Second, laws' generality demands that they make a general claim about the behaviour of things, but since it is plainly false that everything satisfies the behavioural predicates corresponding to all the so-far considered equations, the predication has to be qualified somehow. I have suggested that the most plausible way to do this is by conditioning on a further 'system-predicate' which is satisfied if and only if an entity is of an appropriate type. Hence, an argument has been presented that the laws typically represented by equations are in fact more appropriately rendered in the form of a conditional, in the form $\forall x(S x \rightarrow B x)$, where $S$ is a system-predicate and $B$ a behavioural predicate and both predicates take the same variable.

\subsection{Miscellaneous laws}

We now move on to those laws which superficially, at least, do not appear to be conditionals or equations. The first thing to notice is that the examples given for this 'miscellaneous' group of laws assert the existence of some relationship between variables. Gresham's law relates the variable of quality of currency to time; Buys Ballot's law relates height and direction of pressure with location in hemisphere and direction of wind; Faraday's first electrolysis law relates an element's liberated mass with a quantity of electricity. Whilst not equations as such, they are nonetheless similar in that they describe certain functional relationships.

Similarly to the argument for equations, we can note that these relationships will not be understood properly unless they are interpreted as relating the actual instances of various properties by individual entities. That is, we can infer a 
high-order behavioural-predicate for each putative law which is satisfied by an entity when and only when the relation defined in the functional relation holds between the lower-order properties which feature in that relation. For example, the above expression of Gresham's law indicates a high-order predicate '.. is such that its quantity of undervalued money in circulation is negatively correlated with its quantity of overvalued money in circulation and the quantity of overvalued money is increasing'.

We then also note that a full explication of the law would not predicate such a behavioural property of everything, but rather some type of entity, i.e. we infer an antecedent system-predicate. For Gresham's law, the relevant type of system is clearly a closed economy; for the Buys Ballot law, possibly the Earth itself; for Faraday's first law, a system comprising an electrode and electrolyte. The same argument as above works, therefore, to show the implicit conditional in all the considered laws above. The full rendering in conditional form of Gresham's law would be,

All closed economies are such that their quantity of undervalued money in circulation is negatively correlated with their quantity of overvalued money in circulation and the quantity of overvalued money is increasing.

The argument provided in the previous section seems to extend, therefore, to any law represented as a functional relationship. First, we note that the relationship comprises a predication of something, then we note that the predication must be qualified by a system-predicate if it is to be attributed generally. As predicted, the law turns out to be more fully explicated as a conditional.

\section{Are all laws conditionals?}

I have provided an argument for understanding some of our laws as conditionals. There appeared to be a straightforward way to interpret conditionally the ideal gas law, Ohm's law, Yoda's power law, Gresham's law, Buys Ballot's law and Faraday's first law of electrolysis (among other laws). The argument relied on the understanding that the behaviour described by the functional relationship or equation by which those laws are typically represented is not a behaviour attributed to everything in a relatively unrestricted domain, but rather to a specific class of systems (the ideal gases, the electrical conductors, etc.) which the behaviour is antecedently conditioned on. This style of argument works for the individual cases. However, besides inductive motivation, it does not provide reason to think that every law need incorporate such conditioning.

Indeed, we might think that some of the most austere laws do not need conditionalising in this way, since they apply to everything, unqualified. Consider the physicalist view that physics consists in a project to determine those formulae which most accurately describe the behaviour of every single system in existence by some 
basic formulae and generalisations. ${ }^{11}$ Perhaps the Schrödinger equation is expected to be among those formulae. ${ }^{12}$

$$
i \hbar \partial \Psi / \partial t=\hat{H} \Psi
$$

We might follow the reasoning made above insofar as we recognise the equation to represent some information concerning the behaviour of things, i.e. we identify a behavioural predicate such as '.. is such that its Hamiltonian is related to the timederivative of its wave-function according to the equation $i \hbar \partial \Psi / \partial t=\hat{H} \Psi$.' Let's abbreviate this $S_{t}$. The physicalist may, therefore, believe that there there is a nonconditional law,

$$
\forall x\left(S_{t} x\right)
$$

While such a law may in fact be true, I will argue that it is highly implausible as a rendering of any statement likely to be called a law given the current state of physics. Assuming something like the above is one of the best candidates for a fully general law that the physicalist has to offer, it is not hard to see why no level-headed scientist will be willing to accept it. I offer three points.

First, it is well known that there are technical difficulties extending the current analysis (quantum field theory, quantum chromodynamics, etc.) to large-scale entities. Solutions to the Schrödinger equation, for instance, are just about manageable for Hydrogen and Helium, but even for the immediately more massive atoms, computational methods are required. When we come to consider small molecules or anything more complex, approximation methods like that from Born and Oppenheimer are required in order to make useful predictions or explanations. So, regardless of whether this is considered as mere computational intractability or not, (2) certainly doesn't reflect the practice of contemporary physics. But furthermore, if there is any reason at all to doubt that all macromolecules behave in the way dictated by the $S_{t}$ predicate, any scientist averse to unnecessarily bold conjectures is going to hedge their bets with regard to what the $S_{t}$ predicate applies to. Why commit to saying the $S_{t}$ predicate applies to everything, as (2) does, when one can be far more confident of a weaker claim at no cost of utility: that $S_{t}$ is satisfied by those entities which satisfy some particular antecedent condition? Until our

\footnotetext{
${ }^{11}$ Physicalism does not entail a thesis about reduction (although some physicalists do also hold this thesis). A reductivist will want to claim that entities currently described by other sciences 'reduce', in some sense, to those found in physics. A physicalist need not hold this view. For they might take the other sciences to simply fail to denote anything at all or else have reasons to think that reduction to physics is not a requirement for the ability of physics to describe the behaviour of everything (e.g. a la Davidson 1970).

${ }^{12}$ Schrödinger's equation seems in many respects a paradigm representation of a law (see for example, Maudlin 2007, 11). But it is worth keeping in mind that neither the equation nor the information it represents are typically referred to as 'a law' in scientific practice (this also goes for many equations applied across the sciences). While I expect (as did an anonymous referee) that it is relatively harmless to use it as an example, some have demanded that we take a closer look at the practice of scientific denotation of the title 'law' (e.g. Mumford 2004, esp. p.129).
} 
numerical capabilities are developed enough to derive solutions to the Schrödinger equation (in its unapproximated form) for macromolecules, there is no reason to believe that scientific endeavour supports the commitment to a fully general law like (2) —although we may believe such a law for other (philosophical) reasons.

Second, relativistic quantum mechanics may include the application of Schrödinger's equation to contexts of special relativity, but not general relativity; the latter remains an as yet unresolved problem for physicists (the tensor fields described in Einstein's field equations imply definite values for both position and location of objects whereas the Heisenberg uncertainty principle implies such precision is impossible). As with the problem of computational intractability we may believe a reconciliation between the two theories is possible, but must also admit that there is no current scientific support for laws which entail such a reconciliation. Again, why should a scientist commit themselves to the $S_{t}$ predicate applying to everything when they can be more confident of a weaker claim at no cost of utility? One might nonetheless believe the predicate so applies, but such a belief will not be justified by scientific discovery alone.

Third, even if some reconciliation can be achieved and physics becomes united under one body of theory, we would still be far from justified on the basis of physics alone in our commitment to the view that the theory applied to everything. The project of physics is to describe the behaviour of the physical world: plausibly, that which has location, size, momentum, charge. It is an open question what fits into this ontology; presumably the entities of the standard model and the Higgs particle are there (but what about fields and gravitons?) Whatever we decide, the truth of physics is not hostage to physicalism. The truth of the standard model, for instance, is not thought to stand or fall on the debate over platonism in mathematics or dualism in mind; its truth is independent of these debates. So, physicists' laws and theories must include a restriction of system-type in order to avoid purporting to describe the behaviour of mathematical objects, incorporeal minds, or indeed any type of entity mooted to be irreducible to physical stuff. Note again, that the point here is not that physicalism is false, nor that we have no good reasons to believe it. The point is that we make no explanatory gains by writing unconditional universality into the conjectures of physics. Physics provides some behavioural claims about a particular class of entities, it is up to us as philosophers, biologists, policy-makers, etc. to decide whether or not anything falls outside this class.

I take these points to show that Schrödinger's equation is not applied unconditionally, but rather only to a specific class of systems. It may prove difficult to provide necessary and sufficient conditions for such a system, but the practice of quantum physics will reveal that the class is not typically understood to include general relativistic systems, mental states or platonic forms. It is known to contain systems which can be predicted and explained (potentially including those reasonably approximated) by the formula $i \hbar \partial \Psi / \partial t=\hat{H} \Psi$. Let ' $Q$ ' denote the property of being in such a class of systems. We then get an expression for the knowledge physicists have relevant to applications and interpretations of the Schrödinger equation,

$$
\forall x\left(Q \rightarrow S_{t}\right)
$$




\section{Conclusion and lessons for a subsequent analysis}

I have argued for the need to understand all laws of association as having the deep logical form of a quantified conditional. So, for example, even though we represent Newton's second law of motion with the equation $F=m a$, the law itself is more properly expressed as the conditional:

All Newtonian systems behave in such a way that the value of total force applied to it is equal to the product of its value of mass and its value of acceleration.

Pace Maudlin, I have suggested that the process of putting the information conveyed by an equation like $F=m a$ into the form of a conditional is neither torturous nor useless. In fact, it is all but explicit in the use of the equation and necessary if we are to make sense of science's claims of law.

The failure to make this clear in the philosophical literature is symptomatic of a communicative breakdown between scientists and philosophers of science. The tradition of rendering laws as conditionals was often supported by toy examples like 'all ravens are black', which rightly caused the reaction that it was being submitted without a reasonable scrutiny of genuine scientific theory. When reading about the laws which scientific textbooks and papers convey to us, we have a tendency to focus on the central equations or models being used rather than how the information itself is structured. This can lead us to think a pluralist approach to laws is the most appropriate and condem the project of bringing laws under one logical structure as hopeless. However, I hope I have gone some way to persuade the reader that despite tradition's poor support for it, the conditional rendering of laws is, in central cases, the most appropriate. Equations and other functional relationships may be used to represent laws, but are not laws themselves.

A more detailed characterisation of the conditional form of laws shows that the consequent describes the behaviour of an entity (e.g. its having a qualitative property, its approximation to a model or accordance with an equation). The antecedent describes the type of system from which the law licenses inference to that behaviour. This system predicate may denote a natural kind (e.g. rock strata), an ideal (e.g. an ideal gas, an ideal interface between two media), or a mechanism (e.g. chemical bonding). It may also include so-called 'completer-clauses' (Fodor 1991) to reduce the number of instances which otherwise would falsify the law (e.g. the clause that the system is not operated on by external forces). Admittedly, I do not think that these observations about the system predicate go as far as to rid all laws of all counterinstances - a grand solution to the problem of ceteris paribus laws must be solved elsewhere. Sometimes scientists may have no better way to describe what the system-type is other than by saying that it is that type whose instances behave in the manner of the behavioural predicate. This can make such laws sound trivial. But scrutiny of the practice of the relevant science will reveal a non-trivial understanding of what systems it is appropriate to ascribe the behaviour to. For if there were no implicit understanding, scientists would show no intelligible consistency or method in their application of the behavioural predicate.

I end by end by addressing two points of contact with the wider literature. 


\subsection{System laws}

In some recent discussion on laws, a distinction has been made between 'system laws' and other laws. Schurz, for example, distinguishes 'system laws' from,

...those fundamental laws of physics which are not restricted to any special kinds of systems (be it by an explicit antecedent condition or an implicit application constraint). (2002, 367)

This distinction has the potential for at least two interpretations. According to one, system laws are so-called if they have an antecedent condition which contains a singular term. It seems to be this reading Schurz has in mind when he says shortly after the previous quote that 'system laws [...] refer to particular systems of a certain kind in a certain time interval $\Delta t$ ' (my emphasis). It is in this sense that generalisations found in biology which appear to reference specific domains of time and space (e.g. earth over the last 3.8 billion years) have been distinguished from those more general laws of nature (e.g. in Beatty 1995; Rosenberg 2001). Schurz also mentions Kepler's laws and Galileo's law of free fall, both of which condition on the system of application being, respectively, our solar system and the Earth. (We might also think that Buys Ballot's law counts as a system law for the same reason.)

If this is the correct interpretation, then the above discussion on laws' conditionality is not directly relevant. Although the arguments provided above have aimed to show that laws in general have a conditional form they have not demanded that the antecedent incorporate a singular term, although this might be the case for some laws. However, according to another interpretation, a system law is so-called because it is a conditional, full-stop. This interpretation seems to match with Schurz's other claim that the fundamental laws of physics are not system laws by virtue of not being 'restricted to any special kinds of systems (be it by an explicit antecedent condition or an implicit application constraint)' and his regarding the total force law and special force laws as counting as non-system laws of nature 'at the cost of being per se not applicable to real systems, because they do not specify which forces are active' (Schurz's emphasis). If we read 'special' and 'real' in these quotes as implying that the relevant systems fall under some not-fully-general class, then we have reason to think of any law in the form of a conditional as a system law.

According to the second, broader interpretation, my argument for the conditionality of laws entails that all laws are system laws. The total force law (Newton's second law) and the special force laws (e.g. Newton's gravitational law) are system laws in this sense since they express conditionals, both having the antecedent condition that the system is massive, or perhaps more appropriately 'Newtonian'. The argument for these points drew on the fact that the behaviours these laws describe are not expected to be fully general. Instead, they condition behaviour on entities characterised by a particular body of theory (e.g. classical mechanics) not assumed to capture everything in existence.

Understanding all laws as system laws in this sense allows us to retain their universality, so long as specifying an appropriate, counterinstance-free antecedent is not too troublesome. While it has sometimes been argued that the 'application' of laws 
is restricted to certain models or domains of enquiry (e.g. Giere 1995; Cartwright 1999), it makes less sense to understand system laws this way. Since Frege, it has been commonplace to understand conditionals of the form (1) as not 'applying to' any particular type of thing (e.g. the $F$ s) other than in the sense that they apply to everything in the domain. Hence, if all laws are system laws, and so have the form (1), the notion of application of laws appears to lose significance. In fact, the notion of application has a lot in common with the considered and rejected idea that significant restrictions on universal predication of the behavioural predicate should be made via implicit contextual domain restrictions (see p.10). Domain restrictions require rules of application, possibly specified by contextual parameters. If we deny this as a plausible reading of laws, we simultaneously deny a central reason to stress the importance of the 'applicability' a law.

\subsection{Two predications, one variable}

Philosophers have often assumed (or written as though they have assumed) laws were conditionals but avoided the conclusion of the above arguments that laws exemplify conditionals which operate on two predications of the same variable. For example, Pietroski and Rey say the following. ${ }^{13}$

Science is in the business of providing good explanations. One venerable way it does this is by citing laws from which the eplanandum can be derived [...] Such laws have traditionally been schematized as universal generalizations of the form:

$$
\forall x(F(x, t) \Rightarrow \exists y(G(y,(t+\epsilon))))
$$

where $x$ and $y$ range over objects in some domain; $F$ and $G$ are (perhaps complex) kind predicates of the science in question; $t$ is a time and $\epsilon$ an interval; and ' $\Rightarrow$ ' represents an appropriate connective for nomic conditionals (1995, $82-3){ }^{14}$

There is much in this passage which is in agreement with the above discussion. Pietroski's and Rey's schema has a universal quantifier and, more importantly, is a conditional (although not necessarily material). Arguably, we might even count instances of (1) as instances of the more general (3) in which $x=y$ and $\epsilon=0$ s. This in turn might lead us to think (3) is actually the more appropriate general schema for laws rather than (1). This is a view to which we might attribute Hempel, who claimed that,

... lawlike sentences can have many different logical forms. Some paradigms of nomic sentences, such as 'All gases expand when heated under constant pressure' may be construed as having the simple universal conditional ' $\forall x(F x \rightarrow$ $G x)^{\prime}$; others involve universal as well as existential generalisation as does the

\footnotetext{
${ }^{13}$ There is also a brief discussion of this issue in Armstrong (1983, 153).

${ }^{14}$ Curiously, the $t$ is not bound in the original (thanks to an anonymous referee for pointing this out to me). Assuming Pietroski and Rey do not take $t$ as a constant, the formula needs another universal quantifier phrase ' $\forall t$ ' at the front where $t$ ranges over times.
} 
sentence 'For every chemical compound there exists a range of temperatures and pressures at which the compound is a liquid' (Hempel 1965, 338-9).

In response to Hempel, we might defend schema (1) first by noting that even if his second example doesn't have (1)'s form, Hempel has yet to justify the claim that it is a law. After all, it is not explicitly referred to as a law in science (although it may be explanatory and counterfactually robust). Since our policy of inclusivity was meant to respect scientists ascriptions of the title 'law' to statements and not philosophers' ascriptions, we might choose to simply ignore the example as irrelevant. ${ }^{15}$ But a second, and less close-minded, line of response returns to the lessons learned from the previous discussion. Laws which describe functional relationships between variable properties, as both of Hempel's examples do, are invariably descriptions of the behaviour of instances of a certain system-type. In Hempel's second example, the type of system is chemical compound, where the functional relationship in the consequent is explicitly a feature of instances of that type. Hence, the putative law tells us that there is a range of temperatures and pressures at which the compound mentioned in the antecedent is a liquid. It is possible then to render Hempel's recalcitrant case in the form of (1) where the consequent behavioural-predicate is understood as satisfied by an entity which is such that there exists a range of temperatures at which it is a liquid. Though this may appear to suppress some of the explicit detail of a formulation in which the existential quantifier is explicit, a rendering of the law in the form of (1) makes it clear that, as with any law, the behaviour is attributed to instances of the system-type.

Pietroski's and Rey's schema does not purport to do this, however. In their schema, the consequent attributes a property to an entity potentially distinct and at a different time from instances of the antecedent. Schema (3) seems to be (implicitly or explicitly) the schema in mind in a number of places in the philosophical literature, most notably when laws are employed to analyse causal relations (see, e.g., Paul 2000; Schaffer 2001; Armstrong 2004). Causation is typically taken to be a relation between an earlier cause and a later effect where the two events may involve different entities and since Hume (1738 [1978], 1777 [1993]) it has been commonplace to render laws as constant conjunctions or universally quantified conditionals of the form (3) in order that causal relations may mirror laws' logical structure. Causal relations may thus be understood as successive instantiations of properties $C$ and $E$ in which $C$ appears as the antecedent predicate of a law of the form (3) in which $E$ is the consequent predicate.

Schema (1) cannot mirror causal relations in this way since it says nothing about time differences between property-instantiations and only contains one variable. However, I believe it is nonetheless the appropriate rendering of the kinds of law we have been considering. First, it is unclear to me even what the exact motivation behind schematising laws with (3) is supposed to be. Davidson (1967) pointed out that statements about token causal relations, e.g. 'the short circuit caused the fire', are logically stronger than the assertion of an instance of a conditional 'if there was

\footnotetext{
${ }^{15}$ This comment would also apply to a similar objection to schema (1) from Lowe $(2009,148)$.
} 
a short circuit, then there was a fire'. Since both the events denoted (the short circuit and the fire) must occur in order for former to be true, the former entails but is not entailed by the latter, which can be true if neither occur or only the fire occurs. Hence the claim that token causal relations mirror conditionals in the form of (3) is hard to make sense of. Second, there simply do not seem to be any associative laws of the form of (3). Earlier I distinguished the project of enquiring into the logical form of associative laws from causal laws (where the latter have some causal term or synonym). Presumably those engaged in causal analysis where laws feature in the analysans will want to make use of associative rather than causal laws, otherwise they risk circularity. But at first glance it seems to be only causal laws which have anything approaching the form of (3), where $\epsilon$ can be nonzero and $x \neq y$. Compare Farr's law (above) with Bowditch's law.

Bowditch's law: Any stimulus that will cause a contraction of the heart muscle will cause as powerful a pulsation as any greater stimulus. ${ }^{16}$

Farr's law tells us what epidemics are like. It predicates of a single variable that if it is an epidemic (at a certain time) then it will behave in such and such a way (at that very time). Bowditch's law, however, does predicate of two separate variables: of stimuli and of contractions, respectively. Also, we might understand it as implying that contractions in the heart will occur some time after the stimulus. Hence, Bowditch's law appears to condition the behaviour of a certain type of entity on the earlier behaviour of a different entity, as instances of (3) do. However, Bowditch's law is a causal law and hence not in the range of laws which are being investigated here (see p.2), nor is it in the range of plausible analysans for causal relationships (on pain of circularity).

Upon investigation, it seems arguable that no associative law is sufficient to justify an inference from earlier to later property-instantiations. Associative laws say that things will behave in a certain way so long as they count as an instance of a particular system-type, implying that a system's behaviour is inferable throughout a time only if it remains an instance of the system-type for that time. By contrast, causal laws $d o$ seem to license inferences from earlier to later property-instantiations. We can infer, for example, that a stimulus of the heart sufficient to result in a contraction will be followed by a contraction as powerful as that following any greater stimulus. It is laws like this which capture the fact that instances of stimuli to hearts of certain magnitudes cause instances of contractions of certain magnitudes. But by virtue of being a causal law it is debatable whether or not they explain rather than merely summarise those instances of the causal relation. Moreover, and regardless of whether or not causal laws explain token causal relations, one corollary of the above discussion is that if associative laws are to explain token, non-simultaneous, causal connections, they must be conjoined with further facts, such as the continuing satisfaction of the relevant system-type predicate throughout the duration of the causal relation, in order to justify an inference from cause to effect.

\footnotetext{
${ }^{16}$ This implies that stimuli can cause contractions of the heart muscle only above a certain threshold of magnitude and that all contractions of the heart muscle brought about by such stimuli are as strong as each other.
} 
Acknowledgments I would like to thank Luke Fenton-Glynn, Andreas Hüttemann, Phyllis Illari, Daniel Kodaj and Emma Tobin for their suggestions throughout my development of this paper and the two anonymous referees whose comments were invaluable.

Open Access This article is distributed under the terms of the Creative Commons Attribution 4.0 International License (http://creativecommons.org/licenses/by/4.0/), which permits unrestricted use, distribution, and reproduction in any medium, provided you give appropriate credit to the original author(s) and the source, provide a link to the Creative Commons license, and indicate if changes were made.

\section{References}

Anscombe, G.E.M. (1971). Causality and determinism. Cambridge: Cambridge University Press.

Armstrong, D. (1983). What is a law of nature? Cambridge: Cambridge University Press.

Armstrong, D. (1997). A world of states of affairs. Cambridge: Cambridge University Press.

Armstrong, D. (2004). Going through the open door again. In Collins, J., Hall, N., \& Paul, L. (Eds.) Causation and counterfactuals. Cambridge: MIT Press. chapter 19.

Ayer, A.J. (1999 [1956]). What is a law of nature? In Tooley, M. (Ed.) Laws of nature, causation, and supervenience (pp. 1-52). New York: Garland Publishing.

Bach, K. (1994). Conversational impliciture. Mind and Language, 9, 124-62.

Beatty, J. (1995). The evolutionary contingency thesis. In Wolters, G., \& Lennoz, G. (Eds.) Concepts, theories, and rationality in the biological sciences (pp. 45-81): Pittsburgh University Press.

Beebee, H. (2011). Necessary connections and the problem of induction. Noûs, 45(3), 504-527.

Carroll, J. (2012). Laws of nature. In Zalta, E. (Ed.) The Stanford encyclopedia of philosophy. http://plato. stanford.edu/archives/spr2012/entries/laws-of-nature/.

Cartwright, N. (1979). Causal laws and effective strategies. Nô̂s, 13, 419-437.

Cartwright, N. (1989). Nature's capacities and their measurement. Oxford: Oxford University Press.

Cartwright, N. (1999). The Dappled World: A study of the boundaries of science. Cambridge: Cambridge University Press.

Cohen, J., \& Callender, C. (2009). A better best systems account of lawhood. Philosophical Studies, 145, $1-34$.

Davidson, D. (1967). Causal Relations. Journal of Philosophy, 64(21), 691-703.

Davidson, D. (1970). Mental events. In Foster, L., \& Swanson, J.W. (Eds.) Experience and theory. New York: Humanities Press. chapter, 20.

Drewery, A. (2006). The logical form of universal generalizations. Australian Journal of Philosophy, 83(3), 373-393.

Fodor, J. (1991). You can fool some of the people all of the time, everything, else being equal: Hedged laws and psychological explanations. Mind, 100(1), 19-34.

Giere, R. (1988). Laws, theories, and generalizations. In Grünbaum, A., \& Salmon, W. (Eds.) The limits of deductivism (pp. 37-46). Bekerley: University of California Press.

Giere, R. (1995). Skeptical perspective: science without laws. In Weinert, F. (Ed.) Laws of nature: essays on the philosophical, scientific and historical dimensions (pp. 120-138): Walter de Gruyter \& Co.

Harré, R. (1993). Laws of nature. London: Duckworth.

Hempel, C. (1965). Aspects of scientific explanation. New York: Free Press.

Hempel, C., \& Oppenheim, P. (1948). Studies in the logic of explanation. Philosophy of Science, 15, 135175.

Hume, D. (1738 [1978]). A treatise of human nature: Oxford Clarendon Press.

Hume, D. (1777 [1993]). An enquiry concerning human understanding, 2nd edn. Indianapolis: Hackett Publishing.

Hüttemann, A. (2007). Naturgestze. In Bartels, A., \& Stökler, M. (Eds.) Wissenschaftstheorie (pp. 135153).

Hüttemann, A. (2014). Ceteris paribus laws in physics. Erkenntnis, 79, 1715-1728.

Kneale, W. (1950). Natural laws and contrary-to-fact conditionals. Analysis, 10(6), 121-125.

Lange, M. (2000). Natural laws in scientific practice. Oxford: Oxford University Press.

Lange, M. (2008). Could the laws of nature change? Philosophy of Science, 75, 69-92. 
Lange, M. (2009). Laws and lawmakers: science, metaphysics and the laws of nature. Oxford: Oxford University Press.

Lowe, E.J. (2009). More kinds of being: a further study of individuation, identity, and the logic of sortal terms: Wiley.

Mackie, J. (1973). Truth, probability and paradox: Oxford Clarendon Press.

Maudlin, T. (2007). The metaphysics within physics. New York: Oxford University Press.

Mumford, S. (2004). Laws in nature. Evanston: Routledge.

Nickel, B. (2010). Ceteris paribus laws: generics and natural kinds. Philosophers' Imprint, 10(6).

Paul, L. (2000). Aspect causation. Journal of Philosophy, 97, 235-256.

Pietroski, P., \& Rey, R. (1995). When other things aren't equal: saving ceteris paribus laws from vacuity. British Journal of Philosophy of Science, 46, 81-110.

Reutlinger, A. (2011). A theory of non-universal laws. International Studies in the Philosophy of Science, 25, 97-117.

Rosenberg, A. (2001). How is biological explanation possible? British Journal of Philosophy of Science, $52,735-760$.

Schaffer, J. (2001). Causes as probability raisers of processes. Journal of Philosophy, 98, 75-92.

Schurz, G. (2002). Ceteris paribus laws: classification and deconstruction. Erkenntnis, 57(3), 351-372.

Sellars, W. (1948). Concepts as involving laws and inconceivable without them. Philosophy of Science, $15(4), 287-315$.

Spohn, W. (2002). Laws, ceteris paribus conditions, and the dynamics of belief. Erkenntnis, 52(special issue), 373-394.

Stalnaker, R. (1968). A theory of conditionals, studies in logical theory. American Philosophical Quaterly, $98-112$.

Stanley, J., \& Szabo, Z.G. (2000). On quantifier domain restriction. Mind and Language, 15, 219-261. 